\title{
A Definition of Metastability for Markov Processes with Detailed Balance
}

\author{
François Leyvraz, Hernán Larralde and David P. Sanders \\ Centro de Ciencias Físicas, UNAM; Apartado postal 48-3, \\ CP 62251 Cuernavaca Morelos, Mexico
}

\begin{abstract}
A definition of metastable states applicable to arbitrary finite state Markov processes satisfying detailed balance is discussed. In particular, we identify a crucial condition that distinguishes genuine metastable states from other types of slowly decaying modes and which leads to properties similar to those postulated in the restricted ensemble approach [6]. The intuitive physical meaning of this condition is simply that the total equilibrium probability of finding the system in the metastable state is negligible. As a concrete application of our formalism we present preliminary results on a 2D kinetic Ising model.
\end{abstract}

PACS numbers: 02.50Ga, 05.20.Gg, 05.70.Ln 


\section{INTRODUCTION}

In equilibrium statistical mechanics, it has been accepted for a long time that the canonical ensemble provides, at least for the vast majority of systems, an adequate description of their properties. It could be argued that this essentially reduces the problem of understanding equilibrium properties to one of computation. On the other hand, for statistical mechanics far from equilibrium things are quite different: there are no laws corresponding to the Gibbs ensembles in order to calculate the probabilities of a given configuration in general, and the situation is still extremely complex.

In this respect, metastable states occupy a curiously intermediate position: they are generally viewed as equilibrium states, i.e. as having a well-defined free energy, which is however distinct from that of the corresponding equilibrium state. Nevertheless, it is also clear that such states eventually decay through a process of nucleation which brings the metastable state to a true equilibrium state which is physically quite different.

Traditionally, there have been many attempts to justify associating some equilibrium state to metastability through analytic continuation of the free energy. The oldest of these is, of course, the one due to van der Waals, which indeed works perfectly for mean-field theory. For models with short-range interactions, however, matters are more complicated:

it has been shown [3] that the free energy has an essential singularity at the coexistence curve. Langer [3] provided a way to define an appropriate analytical continuation across the existing cut and gave a clear picture of the cause of the singular behaviour: it is, in fact, due to the presence of the droplets which eventually nucleate the equilibrium phase.

In what follows we pursue a purely dynamical point of view of this problem. That is, we start from the main dynamical features of a metastable state and suggest a reasonable definition in terms of dynamic features alone. We then show that the metastable state thus defined can indeed be viewed as the restriction of the equilibrium ensemble onto a suitably defined subset. To achieve this goal, however, we must pay a (considerable) price: we must assume the system obeys very simplified dynamics, namely Markov chains satisfying detailed balance. Further, we shall concern ourselves strictly with finite systems and shall not take the thermodynamic limit. In part this is due to the fact that real difficulties arise when this limit is taken: since arbitrarily unlikely fluctuations will arise in arbitrarily short times in a sufficiently large system, it turns out that nucleation eventually becomes instantaneous in 
the thermodynamic limit, which is clearly an artefact. A typical way around this problem might be to take a simultaneous limit to systems of infinite size as well as to parameters ever closer to the coexistence curve. We shall instead simply study the finite, though large, system.

The phenomenon of metastability may be described informally as follows (see [6, 7] for a much fuller discussion along similar lines): a system is said to be in a metastable state if, upon starting the system in a certain subset of initial conditions, the system remains for a very long time in this limited subset of the set of all configurations, which is of negligible measure in equilibrium. Further, this subset is macroscopically distinct from the equilibrium state. Also, the return to equilibrium from a metastable state usually occurs in an abrupt fashion, i.e. the macroscopic variables do not change slowly from their metastable values to their equilibrium values, but rather, they remain essentially constant and then suddenly relax to their equilibrium value, by some quick relaxation mechanism. The requirement that the time during which the system remains in a metastable state be "large" means simply that it is sufficient to allow the system to relax to some kind of pseudo-equilibrium state. Thus, in a metastable state, the values of the macroscopic observables of interest will not show any systematic time-dependence, at least after some initial transient, the duration of which is much less than the decay time of the metastable state.

The results presented in this work are derived for generic ergodic, acyclic Markov processes satisfying detailed balance with respect to the Gibbs measure. The typical system we have in mind is the finite kinetic 2D Ising model, which we discuss in section III.

\section{GENERAL FORMALISM}

Let us consider a Markov chain on a finite state space $\Gamma$ with rates $W_{\sigma \rightarrow \sigma^{\prime}}$, where $\sigma$ and $\sigma^{\prime}$ denote states of $\Gamma$. The master equation for the probability of the system being found in state $\sigma$ is given by

$$
\begin{aligned}
\partial_{t} P(\sigma) & =L P \\
L P & =\sum_{\sigma^{\prime}} W_{\sigma^{\prime} \rightarrow \sigma} P\left(\sigma^{\prime}\right)-P(\sigma) \sum_{\sigma^{\prime}} W_{\sigma \rightarrow \sigma^{\prime}} .
\end{aligned}
$$

Since the Markov chain is assumed to be ergodic and acyclic [2], well-known theorems assert that the solution approaches a unique equilibrium $P_{0}(\sigma)$. If we further assume that detailed 
balance holds, that is,

$$
W_{\sigma^{\prime} \rightarrow \sigma} P_{0}\left(\sigma^{\prime}\right)=W_{\sigma \rightarrow \sigma^{\prime}} P_{0}(\sigma)
$$

then it is also well known that the operator $L$ defined in (11) is self-adjoint with respect to the scalar product

$$
(\Phi, \Psi)=\sum_{\sigma} \frac{\Phi(\sigma) \Psi(\sigma)}{P_{0}(\sigma)} .
$$

Since the underlying vector space is finite-dimensional, it then follows that there is a complete orthonormal set of eigenvectors $P_{n}$ satisfying

$$
L P_{n}=-\Omega_{n} P_{n}
$$

where the $\Omega_{n}$ are by definition arranged in increasing order. The existence of an equilibrium distribution implies that $\Omega_{0}=0$ and the corresponding $P_{0}$ is in fact the equilibrium distribution. All other $\Omega_{n}$ are strictly positive.

Using the orthonormality of the $P_{n}$ we can write

$$
\sum_{\sigma} P_{n}(\sigma)=\delta_{n, 0}
$$

implying that $P_{0}(\sigma)$ is normalized and that adding to it arbitrary multiples of $P_{n}(\sigma)$, for $n \geq 1$, does not alter this normalization.

One then arrives using standard techniques [4] at a formal expression for the probability of arriving from $\sigma_{0}$ to $\sigma$ in time $t$ :

$$
P\left(\sigma, t ; \sigma_{0}, 0\right)=P_{0}(\sigma)+\sum_{n=1}^{\infty} \frac{P_{n}(\sigma) P_{n}\left(\sigma_{0}\right)}{P_{0}\left(\sigma_{0}\right)} e^{-\Omega_{n} t} .
$$

We now turn to the characterization of a metastable state within the general setting outlined above. In view of the informal description of metastability sketched in the introduction, it is clear that if we wish to have a behaviour different from equilibrium over a large time scale, one needs that at least one of the $\Omega_{n}$ be much closer to zero than the rest.

Let us assume that $\Omega_{1} \ll \Omega_{n}$ for all $n \geq 2$. Now consider a process evolving from the initial condition $\sigma_{0}$. Then, following (6), in the relevant time range $\Omega_{2}^{-1} \ll t \ll \Omega_{1}^{-1}$, one finds that the configuration $\sigma$ is occupied with the following (time-independent) probability

$$
P(\sigma)=P_{0}(\sigma)+\frac{P_{1}\left(\sigma_{0}\right)}{P_{0}\left(\sigma_{0}\right)} P_{1}(\sigma) .
$$

Note that, due to (5), this is normalized. Since it differs exponentially little from the exact result, we may also conclude that it is everywhere positive, except perhaps in some places 
where it assumes exponentially small negative values; the latter can be fixed by setting the offending negative values to zero and recomputing the normalization.

This result focuses our attention on the value $P_{1}\left(\sigma_{0}\right) / P_{0}\left(\sigma_{0}\right)$, which characterizes the nature of the initial condition. This quantity will be central to understanding the conditions under which the initial condition can truly be called metastable and the resulting probability distribution given by (7) can justifiably be identified with that of a metastable state. Let us be more specific.

In what follows, we denote $P_{1}(\sigma) / P_{0}(\sigma)$ by $C(\sigma)$, and the maximum value of $C(\sigma)$ by $C$. Next we define the two sets $\Gamma_{m}$ and $\Gamma_{e q}$ as follows:

$$
\Gamma_{m}:=\left\{\sigma: \frac{C}{2} \leq C(\sigma) \leq C\right\},
$$

and $\Gamma_{e q}$ is defined to be the complement of $\Gamma_{m}$. The choice of the factor $1 / 2$ to define the lower bound on $C(\sigma)$ in (88) is purely arbitrary and a matter of convention.

We will show that given the previous scenario, the system will have a metastable state, in the sense discussed in the introduction, if

$$
\sum_{\sigma \in \Gamma_{m}} P_{0}(\sigma) \ll 1,
$$

i.e. that the probability of being found in $\Gamma_{m}$ in equilibrium is negligibly small, and we define the "metastable state" as the state described by the probability distribution

$$
P_{m}(\sigma)=P_{0}(\sigma)+C P_{1}(\sigma) .
$$

It should be stressed that, from a physical point of view, condition (99) is the crucial assumption: it allows to distinguish true metastable states from other slowly decaying states. Of course, in concrete instances this hypothesis will not be easy to prove rigorously, and, for this reason, our approach is in a sense somewhat formal. We shall, however, show that a large number of consequences follow from (92). It is therefore sufficient to prove (92) to show that the restricted state approach to the statistical description of metastable states is applicable. (Note that the importance of (9) was already pointed out in [6, 7].)

In what follows we will show that the properties of systems in which assumption (9) holds give rise to a behaviour which can be identified as metastabilty. These properties are:

1. The probability that a state evolving from an initial condition $\sigma_{0}$ for which $C\left(\sigma_{0}\right)=C$ (or very close to it) leaves $\Gamma_{m}$ in a time less than $t$ is of order $\Omega_{1} t$. This justifies 
identifying such a state as a very persistent one. From this result it also follows that

$$
\sum_{\sigma \in \Gamma_{e q}}\left[P_{0}(\sigma)+C P_{1}(\sigma)\right] \ll 1 .
$$

From this inequality and the positivity properties discussed above, we conclude that

$$
P_{1}(\sigma) \approx-C^{-1} P_{0}(\sigma), \quad \sigma \in \Gamma_{e q} .
$$

Note, however, that the above feature is not enough to characterize a metastable state. A slowly decaying hydrodynamic mode, say, would have the same property.

2. The probability that a state is found in $\Gamma_{e q}$ after a time of order $\Omega_{2}^{-1}$, evolving from an initial condition $\sigma_{0}$ such that $C\left(\sigma_{0}\right)=(1-p) C$, is $p$. On the other hand, if the state has remained in $\Gamma_{m}$ for a time of order $\Omega_{2}^{-1}$, then the value of $C\left(\sigma_{t}\right)$ grows to values very close to $C$ on the same time scale. These results are crucial, because they mean that systems which have $C\left(\sigma_{0}\right) \neq C$ relax fast either to equilibrium or to the metastable state. Once they are in the metastable state, they can be described by the probability distribution $P_{m}(\sigma)$ defined in (10). In order to prove this characteristic property, we have to make use of the defining property of metastable states (9).

3. Finally, if we define a new process in which all transition rates connecting the metastable region $\Gamma_{m}$ defined by (8) to $\Gamma_{e q}$ are set equal to zero, we obtain another Markov process, also satisfying detailed balance with respect to the restriction of $P_{0}(\sigma)$ to $\Gamma_{m}$. We show that if both processes are started from the same initial condition $\sigma_{0}$ satisfying $C\left(\sigma_{0}\right)=C$ then the two processes remain close (in the sense of distance in variation) over a time of order $\Omega^{-1}$. This result leads to

$$
P_{1}(\sigma) \approx C P_{0}(\sigma), \quad \sigma \in \Gamma_{m}
$$

and

$$
2 \ln C=\ln \sum_{\sigma \in \Gamma_{e q}} P_{0}(\sigma)-\ln \sum_{\sigma \in \Gamma_{m}} P_{0}(\sigma),
$$

which is interpreted in a natural way as the free energy difference between the two phases.

Note that this final result also allows to carry over standard results valid for equilibrium systems to the metastable case: one first applies the result to the restricted process, 
which is a bona fide Markov process defined on $\Gamma_{m}$ for all times, and then extends it to the metastable case by arguing that the two processes are close for the relevant timescale $\Omega_{2}^{-1} \ll t \ll \Omega_{1}^{-1}$. In particular, the result derived in [1] can partly be rederived in this way: the fluctuation-dissipation theorem holds in metastable states of the kind we describe, because it can be derived as a general property of Markov processes with detailed balance.

The above results therefore indicate that the program of defining a restricted equilibrium ensemble to describe metastability can be carried out in a fairly rigorous fashion in the context of Markovian proceses satisfying detailed balance.

For a detailed derivation of these results, see [4, 5]. Here we content ourselves with a rough sketch of how they come about. Note first the following basic property:

$$
E\left(e^{\Omega_{1} t^{\prime}} C\left(\sigma\left(t^{\prime}\right)\right) \mid \sigma(t)\right)=e^{\Omega_{1} t} C(\sigma(t)) \quad\left(t<t^{\prime}\right),
$$

where $\sigma(t)$ denotes a path of the Markov process defined by (11), and $E$ denotes the expectation value. This relation is easily verified by a straightforward computation and means that $e^{\Omega_{1} t} C(\sigma(t))$ is a martingale.

To prove point (1), assume that the initial condition $\sigma_{0}$ satisfies $C\left(\sigma_{0}\right)=C$. Now (15) means that, on average, $C(\sigma) e^{\Omega_{1} t}$ should neither go up nor down. Since it starts at the highest possible value of $C(\sigma)$, it has nowhere to go but down (on short time scales this is not significantly changed by the factor $\left.e^{\Omega_{1} t}\right)$. Therefore going down a significant amount is unlikely. It is therefore not likely to leave $\Gamma_{m}$ in the relevant time scale.

Point (2) is more technical: it can be shown that the condition (9) implies that $P_{0}$ and $P_{0}+C P_{1}$ are substantially different from zero on two disjoint sets. Therefore, if the initial condition $\sigma_{0}$ satisfies $C\left(\sigma_{0}\right)=(1-p) C$, it evolves into a state given by

$$
\begin{aligned}
P^{(p)}(\sigma) & =P_{0}(\sigma)+(1-p) C P_{1}(\sigma) \\
& =p P_{0}(\sigma)+(1-p)\left[P_{0}(\sigma)+C P_{1}(\sigma)\right] .
\end{aligned}
$$

But this state can be interpreted as being in the equilibrium state with probability $p$ or in the metastable state with probability $1-p$.

For point (3) consider the restricted process, where the rates are defined by

$$
W_{\sigma^{\prime} \rightarrow \sigma}^{R}= \begin{cases}W_{\sigma^{\prime} \rightarrow \sigma} & \sigma, \sigma^{\prime} \in \Gamma_{m} \text { or } \sigma, \sigma^{\prime} \in \Gamma_{e q} \\ 0 & \text { otherwise. }\end{cases}
$$




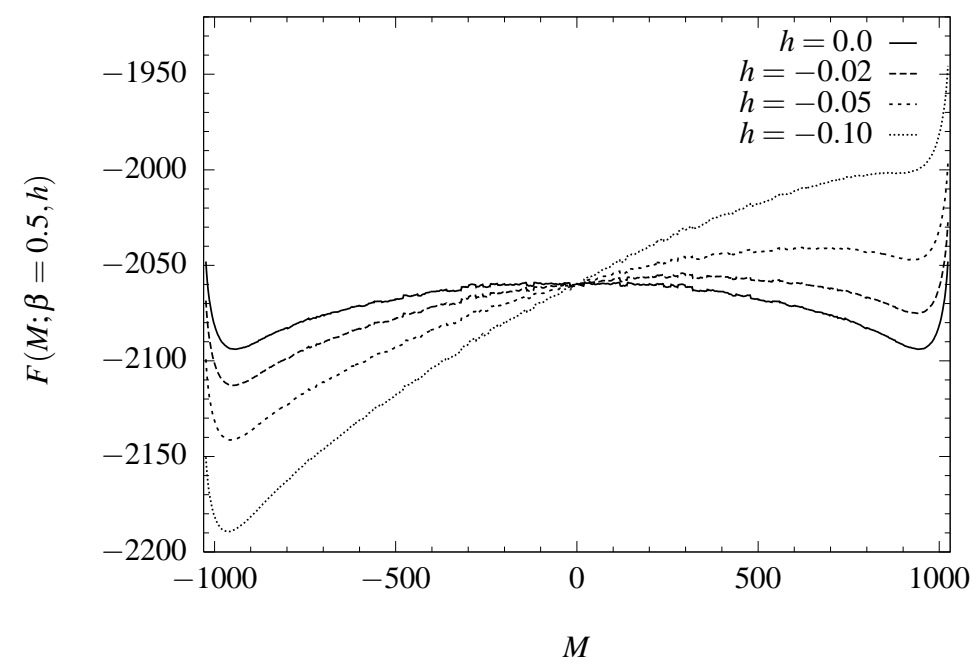

FIG. 1: Free energy $F(M ; \beta, h)$ of the 2D Ising model (see (22) for the definition) for a $32 \times 32$ sample with $\beta:=1 / T=0.5$, i.e. significantly below criticality. Note the secondary minima for $0 \neq|h|<0.05$, which are quite pronounced in spite of our considering a fairly large system.

This process satisfies detailed balance with respect to $P_{0}$, just as does the original process. The trajectories of the physical process starting from $\sigma_{0}$ with $C\left(\sigma_{0}\right)=C$ have the same probabilities as the corresponding trajectories of the restricted process except when the former cross from $\Gamma_{m}$ to $\Gamma_{e q}$. But, as was shown in point (1), such crossings are unlikely, so the two processes indeed remain close to each other in the relevant time range.

\section{AN APPLICATION: THE ISING MODEL}

We now proceed to discuss how these ideas can be applied concretely to the case of the 2D kinetic Ising model. As is well known, if $T<T_{c}$ and a small magnetic field $h$ is applied, then the spontaneous magnetization in equilibrium points in the direction of the field. There is, however, for a broad range of parameters, a metastable state for which the magnetization is in the direction opposite to the field.

We wish to show a way to obtain some confirmation of the ideas described above through simulations of this system. A crucial issue is to identify the observables which play an essential role in determining $C(\sigma)$ and hence $\Gamma_{m}$. For the ferromagnetic Ising model, we assume that these observables reduce simply to the spin interaction energy $E(\sigma)$ and the magnetization $M(\sigma)$. For systems of the size we consider presently, it is not possible for 
a nucleating droplet, which is the crucial factor determining whether a system is or is not about to nucleate, to appear without noticeably affecting the values of $E$ and $M$. We therefore assume that

$$
C(\sigma)=\Phi[E(\sigma), M(\sigma)],
$$

which defines $\Phi(E, M)$.

The equilibrium probability of a configuration $\sigma$ is

$$
P_{0}(\sigma) \propto \exp [-\beta E(\sigma)+\beta h M(\sigma)],
$$

where $\beta:=1 / T$. Summing over all configurations $\sigma$ having the same value of $E$ and $M$, the equilibrium probability that the system is in the macrostate $(E, M)$ is

$$
P_{0}(E, M) \propto g(E, M) \exp [-\beta(E-h M)] .
$$

Here, $g(E, M)$ is the density of states of the Ising model, given by

$$
g(E, M)=\sum_{\sigma} \delta[E-E(\sigma)] \delta[M-M(\sigma)] .
$$

This can be computed numerically in an efficient manner, say using the Wang-Landau algorithm [9, 10].

In the metastable region $\Gamma_{m}$ one has $P_{m}=P_{0}+C P_{1}=\left(1+C^{2}\right) P_{0}$, so that $P_{m}$ can also be expressed in terms of $E$ and $M$ using (20). Since the metastable state is well characterized by specific values of $E$ and $M$, it is to be expected that the expression on the r.h.s. of (201) will show a local maximum at these non-equilibrium values. This is confirmed numerically for $P_{0}(E, M)$ as a function of $E$ and $M[5]$.

Here we plot in Figure 1 the free energy

$$
F(M ; \beta, h):=-\frac{1}{\beta} \ln \sum_{E} g(E, M) \exp [-\beta(E-h M)]
$$

at a fixed subcritical temperature. A secondary minimum is seen in Figure 1, which corresponds to the metastable maximum of $P_{0}$. Such a state of affairs will not exist in the thermodynamic limit: indeed, up to an additive term $h M, F(M ; \beta, h)$ is the free energy of the Ising model in an ensemble of fixed temperature and magnetization. By standard theorems on short-range systems [8], this must be equivalent in the thermodynamic limit to the free energy computed as the Legendre transform of the Gibbs potential calculated in the 
grand canonical ensemble. Hence, as the thermodynamic limit is approached, $F(M ; \beta, h)$ must tend to a convex function in $M$, and the additive term $h M$ does not alter this fact. This is clearly at odds with the presence of two minima in $F(M ; \beta, h)$ as shown in Figure 1. Therefore, our identification of $E$ and $M$ as adequate variables to determine $C(\sigma)$ is not tenable beyond a certain sample size. This is in agreement with the fact that for large systems a nucleating droplet may appear without affecting the values of $E$ and $M$.

In the systems in which $E$ and $M$ do furnish a complete description, it is possible to determine $C$ and hence the free energy difference betwen the stable and metastable phase as follows: (14) yields $C$ as a function of the probability of finding the system in $\Gamma_{m}$ in equilibrium. Since we have identified $\Gamma_{m}$ with a certain part of $(E, M)$ space, we can readily compute this probability once $g(E, M)$ is known. This free energy difference can also be compared with one obtained from hysteresis curves, and the comparison is quite satisfactory. These results will be discussed more extensively in 5]. Finally, extensions of this formalism to systems characterized by having several metastable states appear possible and are also presently under way.

\section{Acknowledgments}

The support of DGAPA project IN100803 is gratefully acknowledged. Incisive comments from P. Hänggi, which contributed to the authors' understanding of the role of the thermodynamic limit, are also sincerely appreciated.

[1] G. Báez, H. Larralde, F. Leyvraz and R.A. Méndez-Sánchez, Phys. Rev. Lett. 90, 135701 (2003)

[2] P. Brémaud, Markov Chains, Gibbs Fields, Monte Carlo Simulations and Queues, Texts in Applied Mathematics Vol. 31 (Springer, New York, 1999)

[3] J.S. Langer, Ann. Phys. (N.Y.) 41, 108 (1967)

[4] H. Larralde and F. Leyvraz, Phys. Rev. Lett. 94, 160201 (2005)

[5] H. Larralde, F. Leyvraz and D.P. Sanders (in preparation)

[6] O. Penrose and J.L. Lebowitz, J. Stat. Phys. 3, 211 (1971) 
[7] O. Penrose and J. L. Lebowitz, in Fluctuation Phenomena, edited by E. W. Montroll and J. L. Lebowitz, Studies in Statistical Mechanics (North Holland, Amsterdam 1979), Vol. 7

[8] D. Ruelle, Statistical Mechanics: Rigorous Results, World Scientific, Imperial College Press (1999)

[9] F. Wang and D.P. Landau, Phys. Rev. Lett. 86, 2050 (2001)

[10] F. Wang and D.P. Landau, Phys. Rev. E 64, 056101 (2001) 